\title{
The control of tuberculosis: a continuous game of snakes and
}

\author{
T Ramakrishnan* and P Chandrasekhar ${ }^{\dagger}$ \\ Department of Microbiology and Cell Biology, Indian Institute of Science, Bangalore 560 012, India \\ ${ }^{\dagger}$ National Tuberculosis Institute, 8, Bellary Road, Bangalore 560 003, India \\ *Corresponding author (Email,tr@mcbl.iisc.ernet.in).
}

\begin{abstract}
'The traditional Indian game of snakes and ladders is played by rolling dice to determine how many squares a player can move his marker up the board, starting at square one and finishing at square one hundred ... [there is] an austere geometry of squares broken by angled ladders and snakes with yawning jaws. Landing at the foot of the ladder meant you could climb it, sometimes moving thirty squares in a single throw. You could also make it to square ninety nine, only to encounter a snake .... Then you had to slide down the serpent'.
\end{abstract}

Gita Mehta

"Snakes and Ladders"

1.

\section{Introduction}

Tuberculosis (TB), which had been controlled to a significant extent during the nineteen sixties and seventies, has re-emerged in recent years as one of the leading causes of death in the world. Nearly 3 million people die every year due to TB. A World Health Organization report of 1997 states that there are, world-wide, 8.8 million new cases every year - meaning about 1,000 new cases every hour. Most of these are resistant to the presently available antitubercular drugs. This works out to 52,000 deaths per week, or 7,000 deaths each day and constitutes a grim reminder of the apparent failure of medical science to counter an ancient scourge. Tuberculosis got its present name at the end of the 19th century. Under its previous name of consumption it has been long known as a world-wide phenomenon. It is caused by a bacterium, Mycobacterium tuberculosis. A related organism, $M$. bovis, causes TB in cattle while $M$. leprae is the causative agent of leprosy in man. The non-pathogenic mycobacterium $M$. smegmatis is comparatively safe to work with and is often used in the laboratory as a model to understand the physiology of the members of the entire genus since it grows more rapidly in liquid culture in the laboratory than the pathogenic organisms. (M. tuberculosis takes 15-21 days for optimal growth in stationary liquid culture.)

Through the ages, the contribution of TB to the misery of mankind has been immeasurable. About 70 years ago, as small boys, the authors of this article underwent the traumatic experience of seeing a very close relative (in one case the mother and in the other an aunt) suffering from TB. In those days the only treatment available was a normal diet supplemented with calcium tablets, cod liver oil and an occasional injection of gold suspension. Until the advent of independence in 1947 and the more or less simultaneous beginning in the use of streptomycin, there was no generally available treatment for TB in India. Rest in a sanatorium situated in a salubrious place with an unpolluted atmosphere was strongly recommended but not always possible. When the racking coughs and the accompanying fever become severe, surgical intervention was sometimes attempted. This could take the extreme form of thoracoplasty (removal of ribs) for the closure of cavities, or removal of one of the lungs. Often a merciful death intervened. 
In many respects we live in a different world today. While normally reliable drugs for the treatment of TB are at hand, the proliferation of resistant strains and the association of TB and AIDS are beginning to pose major problems. The recent deciphering of the complete genomic sequence of M. tuberculosis (Cole et al 1998) has given a major impetus to attempts to tackle the disease by using the techniques of modern molecular biology and immunology. The significance of knowing the

DNA sequence of $M$. tuberculosis can hardly be exaggerated, for it is a matter of urgency to devise new therapeutic and prophylactic measures. At the same time, we need to acknowledge that in the past, major successes in the fight against infectious diseases (including TB) have come, not so much from new drugs as from improvements in nutrition and the adoption of public health measures such as better sanitation, provision of safe drinking water, isolation of symptomatic individuals and so on. Records from many countries testify to this (figure 1). Where do we stand today in India with regard to the fight against TB? The aim of this essay is to provide a personal perspective of TB-related research in post-independent India, an evaluation of the work done so far and a peep into the future. We do so from two points of view, those of basic science (TR) and medicine (PC). Most references to the literature prior to 1994 will be found in Ratledge and Stanford (1982), Ramakrishnan (1983) or Bloom (1994).

\section{Tuberculosis in India: From tragedy to optimism}

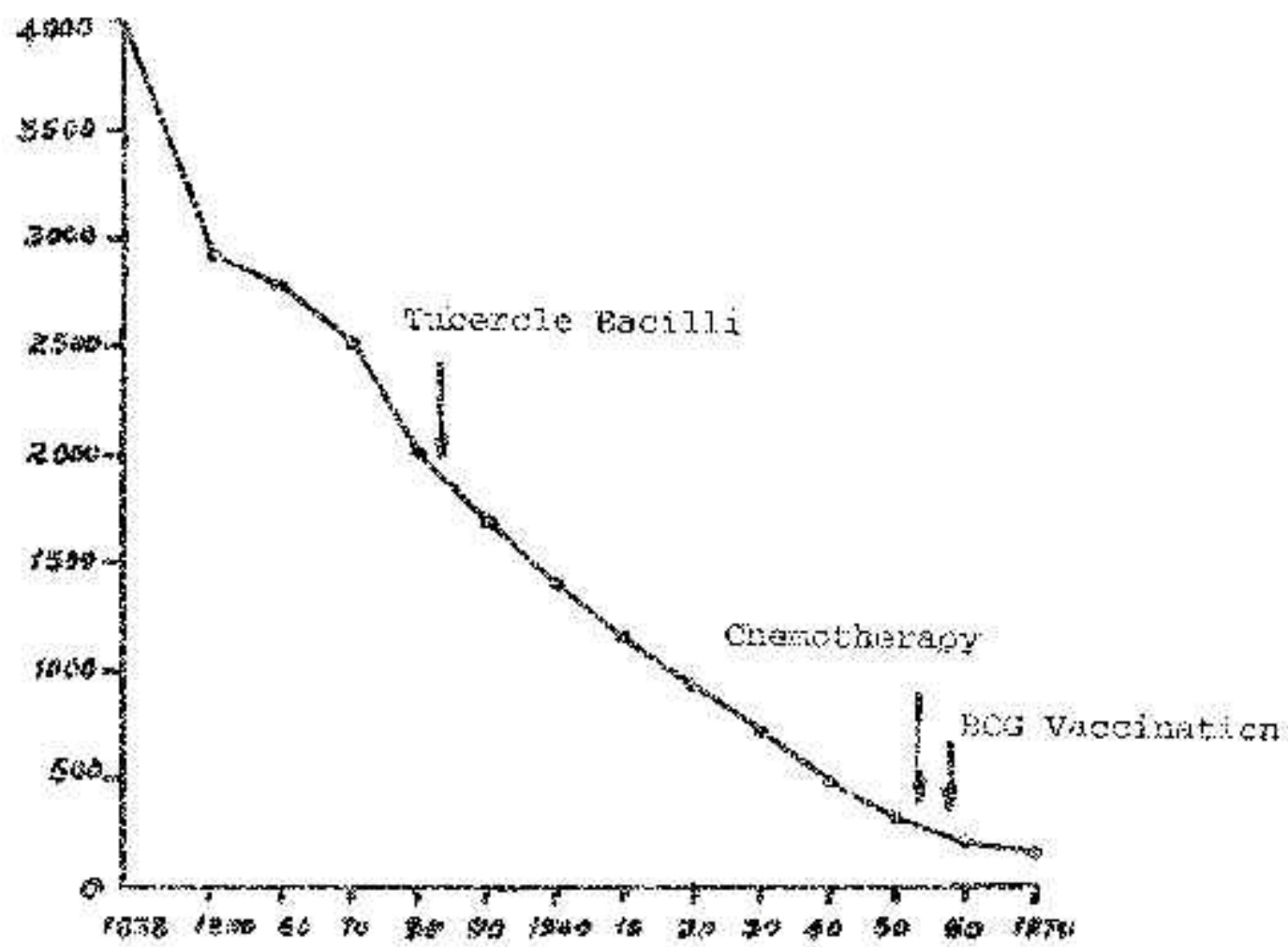

TB has been described as far back as $600 \mathrm{BC}$ in the Sushruta Samhita, a compendium of ancient medicine and surgery. In Sanskrit the disease is known as Kshaya, 'wasting disease', or Raja Yakshmaa, 'the king of diseases'. The second name may be related to the fact that among its sufferers was said to be the moon, the king of stars. There were said to be four causes of the disease: overstrain, suppression of natural urges, wasting (for example, due to grief, anxiety or longing) and a promiscuous diet, any of which could cause the three morbid humours Vata, Pitta and Kapha to flare up. The premonitory symptoms ranged from 'pallor of the eyes' to 'finding fault 
in the service of dinner'; a person with a full-blown case of Raja Yakshmaa could suffer from 'fulness of the head', 'cough', 'pain in the sides', and so on. A treatment based on the principles of Ayurveda, the classical Indian system of health and healing, was provided. Besides medicines, dietary prescriptions were detailed; alcohol in moderate quantities, the flesh of birds and animals which inhabit dry areas and goat's milk were among the items recommended. Some sages were of the opinion that the disease could be spread from person to person and listed it in the class of communicable diseases along with leprosy, fevers, conjunctivitis and syphilis. All said and done, TB was rare until the second half of the nineteenth century. Concomitant with the growing popula-tion density caused by industrialization, its incidence has increased progressively since then (Wilkinson 1914).

One of the earliest sanatoria in the country was established in 1915 in Madanapalle in the state of Andhra Pradesh. Among the persons treated there during the 1940s - pre-chemotherapy days - was PC's aunt. After visits during the summer months for several years, the physicians at the sanatorium recommended thoracoplasty. She did not survive the operation. The emotional strain caused by this episode eventually led PC to decide on taking up TB as his clinical speciality. Happening to become a staff member of the very same sanatorium in 1955, he reviewed the case history and X-rays of his aunt and was astonished to discover how long she had led an active and cheerful life in spite of gross pathology in the lungs.

Ever since Koch (1932) isolated M. tuberculosis in 1882 and demonstrated that it was the aetiological agent of the disease, efforts were under way to control the growth of the organism. Calmette and Guérin isolated an attenuated culture of $M$. bovis, a member of the tuberculosis complex, named Bacillus Calmette-Guérin (BCG). BCG was shown, first in animals and then in neonates, to function as a vaccine against TB. BCG is even now administered to infants, but it has been found that protection is confined to about 10 years and is effective only during childhood. In spite of the availability of potent antitubercular first-line drugs like isoniazid, pyrazinamide and rifampicin, and a number of second-line drugs added every year, the incidence of TB in India and worldwide has risen alarmingly in recent years.

By 1900 it had become clear that the Western method of dealing with the problem of TB - namely, training of specialists, increasing the number of beds and 'running several vehicles', often in different directions - was inappropriate to the Indian situation, which was characterized by meagre resources. In spite of this realization, a properly thought-out national TB control programme (NTP) that combined elements of prevention (BCG vaccination), establishment of clinics, rehabilitation centres and basic research had to await the recommendations of the Bhore committee in 1945. The names of those who provided leadership in the early days of the programme deserve to be remembered: P V Benjamin, architect of the NTP; J Frimodt Möller, responsible for the first BCG trial in the country; B K Sikhand, who vigorously advocated domiciliary treatment and home care; Rajnarayan and S P Pamara, both epidemiologists; M D Deshmukh, who favoured a camp approach; and P K Sen, who studied how the infection was transmitted between individuals living or working in close proximity to one another. All of them shared an extraordinarily strong committment to their goals.

One of the steps taken right at the beginning was the mass BCG campaign launched in 1951. For the first time a strategy was evolved to reach rural areas and it had a good deal of success. The NTP was born in 1962 - it was a people-oriented programme, with the concepts of integration and the principles of primary health care built in and involving the health services of the entire country. It went on to be scientifically tested and implemented in two districts of the country, Tumkur in Karnataka and Ananthapur in Andhra Pradesh. The aim of the NTP was to cut the transmission of the infection through proper and adequate treatment. The focus was on identifying those who excreted tubercle bacilli in their sputum. A study showed that the yield from the first two specimens 
was $80 \%$; additional specimens did not contribute anything substantial. Diagnosis of non-pulmonary TB (which is non-infectious and constitutes $15-20 \%$ of all cases) was by a two-referral system. There was no attempt to consider eradication of the disease, since TB - unlike, for example, small pox - does not qualify for the criteria necessary for eradication. The main problems with regard to TB are that (i) there is a long incubation period, (ii) both subclinical and clinical cases occur; (iii) reinfection and reactivation of latent infections play a prominent role in the development of disease, (iv) the effectiveness of vaccination is still uncertain, BCG being effective only during childhood, and (v) immunity is either partial or doubtful (cell-mediated immunity is unstable; it can be lowered in acute viral infections, during treatment with cytotoxic drugs and steroids, metabolic disorders like diabetes and AIDS). These factors also make it unlikely that an effective biomarker for TB can be discovered in the near future. At any rate, no reliable serodiagnostic test is currently available for extra-pulmonary TB (Chauhan 1997).

As with many progressive measures in this country, in actual implementation NTP did not prove to be a success. This is evidenced by the fact that in the nearly 40 years since its inception the number of TB positives in the country has actually increased (table 1), albeit with phases of decline now and then. The reason for this soon became clear. NTP depended completely on keeping the patient on a four-drug regimen continuously till his sputum became free of microorganisms. The advantages of this regimen are two-fold. Firstly, it prevents the emergence of drug-resistant strains of $M$. tuberculosis. Secondly, since most of the anti-TB drugs are hepatotoxic, a four-drug regimen permits the lowering of the dose of individual drugs to a level at which their hepatotoxicity is low. In practice this criterion was often never fulfilled. Many private physicians prescribed only one drug at a time and changed over to a new one when the first proved no longer effective. Patients tended to stop taking drugs once they started feeling better. The result was that after an initial period of improvement, many patients started harbouring $M$. tuberculosis strains that were resistant simultaneously to several drugs and they also infected healthy people. The situation has improved slightly after 1997 when WHO introduced the DOTS (directly observed therapy short course) system in which patients are closely monitored continuously for six months. However, statistics from a study in Baku, Azerbaijan (reported in Lancet 353 969-973, 1999) show that only $71 \%$ of those who completed DOTS were cured, many of the patients turning out to have harboured drug-resistant strains before the start of the treatment (a clear distinction between relapse and re-infection in such patients is yet to be established). To sum up, the need to develop new drugs against $M$. tuberculosis remains an important one; and basic research on the biochemistry and molecular biology of the organism is essential if we are to have any hope of doing so.

\section{Metabolism and chemotherapy: Beginnings}

The XIV International Tuberculosis Conference was held for the first time in India in New Delhi in 1957 under the presidentship of P V Benjamin, Advisor to the Government of India and architect of the National TB Programme. One of us (TR) attended the conference and in the same year started work on the metabolism of $M$. tuberculosis $\mathrm{H}_{37} \mathrm{Rv}$ and other mycobacteria in the then Pharmacology Laboratory of the Indian Institute of Science, Bangalore. M Sirsi had set up facilities there for testing on tubercle bacilli, grown both in vitro and in experimental mice and guinea pigs, compounds synthesized in the Department of Organic Chemistry by

$\mathrm{B} \mathrm{H}$ lyer and his group.

The work that was initiated in 1957 had three aims: (i) To study the enzyme systems of virulent bacilli and compare them with those reported in the human host, so that any differences could be exploited for a possible chemotherapeutic attack against TB; (ii) to compare the enzyme systems of the drug-sensitive and drug-resistant strains and to study the mechanism of resistance to drugs such as isoniazid and streptomycin, in order to combat the increasing incidence of drug-resistance in India; and (iii) to compare the enzyme systems of virulent and avirulent strains to find out the 
possible cause of virulence.

Compared to the situation at present, during the early years after independence research in India had to be carried out under somewhat demanding circumstances. Two examples may suffice to drive home the point. It was difficult to obtain even a standard biochemical like adenosine triphosphate (ATP); the worker had to prepare it from rabbit muscle after anaesthetizing the animal by repeated injection of magnesium sulphate solution into the ear vein and isolating its muscle. Another common situation was that it often became necessary to calibrate capillary glass tubes with mercury because micropipettes were not readily available in the country. Apart from overcoming such hurdles, until 1961 the work had not yielded any significant results on marked differences between the enzyme systems of the virulent and avirulent strains of mycobacteria, on the one hand, and between virulent mycobacteria and a commonly occurring bacterium like Escherichia coli on the other.

In that year, a fortuitous meeting at Delhi with Jacques Monod convinced TR that only the application of the newly emerging area of molecular biology could clarify the mechanism of virulence and drug resistance of $M$. tuberculosis $\mathrm{H}_{37} \mathrm{Rv}$. At the time the obvious place to learn the techniques connected with this discipline was the USA. TB, however, had ceased to be a menace there and there was no prominent laboratory in the USA carrying out research on the disease (a situation that prevailed until the emergence in the 1980's of AIDS and its attendant complications). The necessary techniques had to be learnt while working on $E$. coli - in the universities of Yale, California (Berkeley) and Michigan - with the hope of adapting them later to M. tuberculosis.

\section{Anti-tubercular drugs}

The ever-growing emergence of strains of $M$. tuberculosis resistant to presently-available drugs has made the control of TB, especially in India and other developing countries, a difficult proposition. Drug-resistant strains appear initially in patients who are not adequately treated with a combination of three or four drugs simultaneously. (The drugs normally used in combination are isoniazid, rifampicin, ethambutol and pyrazinamide.) Later, because of direct infection via the patient, these strains are also found in persons who have never been treated. This situation has necessitated the search for newer antitubercular drugs to which the strains are susceptible. One method to accomplish this is to make use of the knowledge about the mechanism of action of presently available drugs and chemically synthesize drugs which inhibit the metabolic activity of the organism.

Following its discovery by Waksman in 1944 during the early years of the antibiotic era, streptomycin was demonstrated to be an effective antitubercular agent. The discovery of other powerful antitubercular drugs such as isoniazid $(\mathrm{INH})$, ethambutol, pyrazinamide and rifampicin followed. These were mostly discovered by accident and worked by influencing different stages in the metabolic pathways of $M$. tuberculosis; the elucidation of their precise mode of action would lead to the rational development of newer drugs, notably derivates of rifampicin. For a while, the incidence of TB declined dramatically, especially in the industralized countries.

The strain of M. tuberculosis which has been used for work on metabolism has been the $\mathrm{H}_{37} \mathrm{Rv}$ strain originally isolated in 1934. Unlike many clinical isolates, this strain has uniquely retained full virulence in animal models of TB. $\mathrm{H}_{37} \mathrm{Rv}$ is also susceptible to antitubercular drugs and amenable to genetic manipulation. Because $M$. tuberculosis loses its virulence following prolonged passage on Dubos slants, the preferred solid medium for its growth, $\mathrm{H}_{37} \mathrm{Rv}$ and its non-virulent counterpart were obtained after passage of the less virulent variants through experimental mice. These were designated as $\mathrm{H}_{37} \mathrm{Rv}$ and $\mathrm{H}_{37} \mathrm{Ra}$ respectively. However, the strains were not frozen until 1940, and thus the original clones have been lost. Moreover, $\mathrm{H}_{37} \mathrm{Rv}$ loses its virulence on repeated 
subculturing; and has to be passed through experimental mice and tested for its pathogenicity. As mentioned, it is this strain whose genomic sequence has now been deciphered. One hopes that its pathogenicity was confirmed before the sequence study was carried out.

The catalase-peroxidase complex of the organism is a site in the metabolic pathway of $M$. tuberculosis at which drugs could be targeted. Isonicotinic acid hydrazide or isoniazid (INH) is a molecule of simple structure which was discovered in 1951, and found to be a potent antitubercular agent. Even now it is the least toxic of the drugs used for this purpose. Its mode of action has been under investigation from 1957 at the Indian Institute of Science. After a few apparently misleading starts, it was discovered that the drug acts on the catalase-peroxidase complex and the organism loses this enzyme activity when it becomes isoniazid-resistant (Gayathri Devi et al 1974, 1975). This finding was presented by TR at the International Colloquium on the Genetics of Actinomycetales held at Forschungsinstitut Borstel in Borstel, Germany, in 1976 (figure 2). It was proposed that in the presence of catalase-peroxidase, isoniazid is converted to a toxic compound which inhibits the growth of M. tuberculosis (Ramakrishnan 1983). A structure of the postulated compound was suggested on the basis of its molecular weight. No attempt was made, however, to suggest the site in the bacterium on which this compound acts. On hindsight, this was unfortunate. When these results were presented at the committee on Drug Development of WHO at Geneva in October 1983, it was also suggested that any other compound which inhibited the activity of catalase-peroxidase and belonged to the category of heme-dependent enzymes known as hydroperoxidase I could act as an effective agent against INH-resistant organisms. The added advantage of such drugs is their specificity to mycobacteria; they do not inhibit the growth of other microorganisms. Zhang et al confirmed the catalase peroxidase site of the drug and further showed that any mutation at this site (KatG) makes the organism resistant to INH. On the other hand, Winder and Collins (1970) presented evidence to show that INH inhibits mycolic acid synthesis in the cell wall lipid metabolism of $M$. tuberculosis.

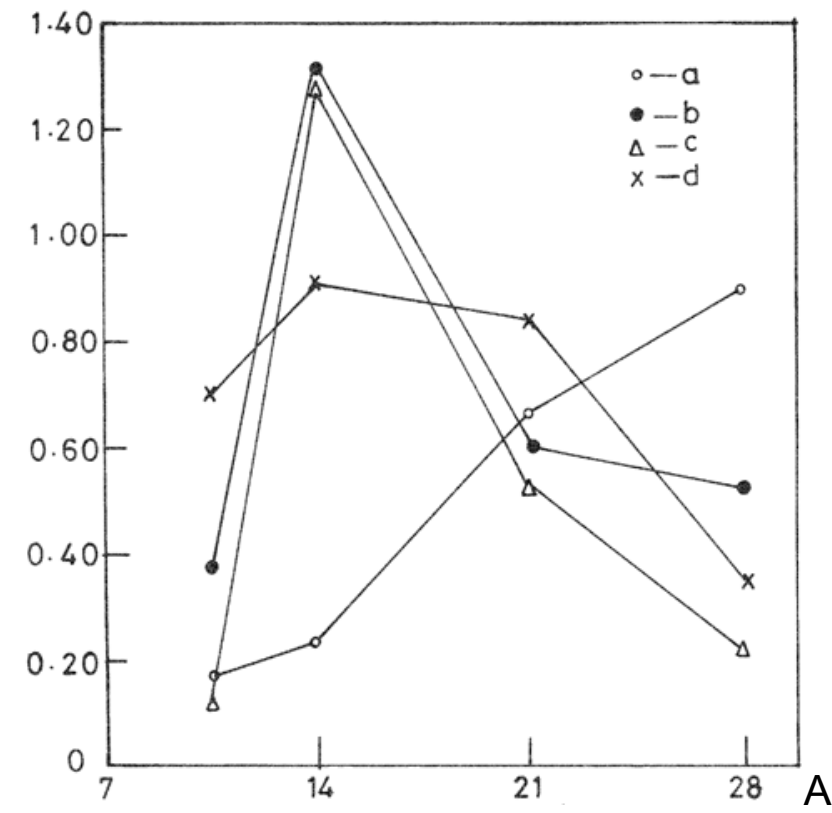

possible explanation of the apparent contradiction between the results of two groups was proposed by Winder (1982) who suggested that catalse-peroxidase may be involved in the chemical modification of INH which destroys some essential components of the bacteria. In support of this, it has been shown recently (Rozworski et al 1998) that INH directly targets a long chain enoyl-acyl carrier protein reductase, an enzyme essential for mycolic acid biosynthesis in $M$. tuberculosis; and further, that incubation of $M$. tuberculosis with radio-labelled $\mathrm{INH}$ results in the accumulation of an $80 \mathrm{kDa}$ protein complex (InhB) containing two proteins, AcpM and KasA (Heym 1999). AcpM is a carrier protein for long chain lipids and KasA is a b -keto-acyl AcP synthase; both proteins participate in the synthesis of 
meromycolic acids. Thus the action of INH is on lipid synthesis after all, with inhibition affecting the formation of the mycobacterial cell wall. M. tuberculosis contains a diversity of lipids and contains some 250 distinct enzymes involved in fatty acid metabolism. In contrast, E. coli has only 50 (Riley and Labadan 1996). It may therefore be worth looking for inhibitors of key steps in lipid metabolism in $M$. tuberculosis as possible antitubercular drugs, especially since mycobacteria contain novel lipid components (Mennikin 1982). It is pertinent to note that ethionamide, a second line antitubercular drug, acts directly on the synthesis of mycolic acids (Winder and Rooney 1970) at a site which has been shown recently to be $b$-ketoacyl synthase.

Among the possibilities for using particular enzymes as targets for drug action, it may be profitable to search for inhibitors of isocitrate lyase, a member of the glyoxylate pathway of M. tuberculosis. This enzyme, which is not essential for the human host, and in H37Rv links carbohydrate metabolism to lipid metabolism, is the only tricarboxylic acid cycle-related enzyme whose activity is increased during the aging of the bacterial cells (figure 3). This stage is reached during anaerobic conditions under which lipids are synthesized by the organism to protect itself from macrophages. The virulent strain is better adapted for survival under low oxygen tension than the avirulent strain. The increased activity of isocitrate lyase upon aging is not observed in the avirulent strain or in $M$. smegmatis (Seshadri et al 1976). At least one group, in the Tuberculosis Research Laboratory in Long Beach, California, has been working on the glyoxylate pathway enzymes as possible sites of drug action (L G Wayne, personal communication).

It is surprising that penicillin and its derivatives, as also synthetic penicillins, have not been adequately tested for their inhibitory action against $M$. tuberculosis. The acid-fastness of the organism, which makes its identification by the Ziehl-Neilsen method possible, tends to eclipse the fact that mycobacteria are also Gram-positive. Penicillin is the only antibiotic known which is specifically active against Gram-positive microorganisms. This makes it worth trying whether one or more compounds related to penicillin can inhibit the activity of $M$. tuberculosis either by themselves or along with the three drugs normally used simultaneously to treat cases of TB. The technique of re-engineering penicillin to produce an antibiotic which is active both against drug-sensitive and drug-resistant bacteria (Rosen et al 1999) appears to be a promising approach for developing a new drug.

\section{Molecular biology-based approaches}

Work on the molecular biology of $M$. tuberculosis required some time to take off because of vigorous opposition of biochemists to this new discipline. Earlier in the century biochemists had suffered at the hands of organic chemists who were opposed to recognition being accorded to biochemistry as a separate subject. Now they were reacting, in their turn as it were, in the same way to molecular biology. Fears were expressed that the introduction of molecular biology could destroy biochemistry in the Indian Institute of Science. The syndrome is not uncommon; physicists have exhibited it too. (One hopes that molecular biologists, when faced in the future with another emerging discipline, will not react similarly.) These teething troubles notwithstanding, the work got going. The situation finally resolved itself when the brightest of the young persons who sought admission to the Biological Sciences departments of the Indian Institute of Science insisted that they be allowed to work for a Ph D in the area of Molecular Biology. As Bertrand Russell says in his autobiography: "Generally, there is old science, which is official, and new science, which elderly men look upon with horror. This results in a continuous battle between old men, who admire the science of their fathers, and the young men who realise the value of their contemporary work".

The importance of the transcriptional machinery in the complexity of the choices faced by $M$. tuberculosis is reflected in the fact that in the genomic map of the organism 13 putative sigma factors and more than 100 regulatory factors of transcription have been predicted (Cole et al 1998). 
Not surprisingly, inhibitors of transcription such as rifampicin are potent drugs. Indeed, the RNA polymerase of $M$. tuberculosis, specifically its sigma subunit, is 1000 times as sensitive to this drug as the E. coli enzyme (Harshey and Ramakrishnan 1976). A search for other compounds with a comparable mode of action resulted in the finding that $\mathrm{N}$-[2-naphthyl] glycine hydrazide inhibits the growth of M. TB by inhibiting the action of RNA polymerase. (Rekha Prabhu et al 1986). Unfortunately, the drug binds to serum proteins, and when tested in experimental mice was found to be needed in large doses for a significant effect. It should be possible to obtain similar inhibitors of RNA-polymerase with decreased binding to serum protein. Yet another approach towards inhibiting the growth of $M$. tuberculosis is to target its translation machinery, specifically poly U-directed protein synthesis. Streptomycin and kanamycin act in this manner, causing misreading of codons and thus inhibiting the translation process. The authors hope that with the new information available from the sequence of the genome of $M$. tuberculosis, newer methods for disease control will become available. Curiously, though, genome analysis has not revealed the existence of classical virulence factors such as toxins or classical invasins.

Another promising target for inhibition of growth of $M$. tuberculosis is DNA topoisomerase. There are two distinct classes of topoisomerases - type I and type II. DNA gyrase, a type II topoisomerase, could be an ideal target for drug design. The antimycobacterial drug norfloxacin acts on the DNA gyrase-DNA complex and inhibits DNA and RNA synthesis of the organism Interestingly, norfloxacin belongs to the group of fluoroquinalones which were discovered while searching for drugs against other bacterial infections (caused by Pseudomonas, Mycoplasma, E. coli and Clostridium). Another inhibitor, novobiocin, which has yet to be clinically tested, inactivates the DNA gyrase $B$ subunit, again inhibiting DNA and RNA synthesis. Recently gyr A and gyr $B$ have been cloned from M. tuberculosis and M. smegmatis. A stretch of 165 amino acids found in $E$. coli gyr $B$ is absent from mycobacterial gyr $B$ and thus any drug acting against the latter would be uniquely specific to mycobacteria (Madhusudan et al 1994; Madhusudan and Nagaraja 1996).

The proliferation of tubercle bacilli is necessary for the successful establishment of a primary infection and the subsequent invasion of the host. This makes the cell division proteins $\mathrm{FtsH}$ and FtsZ useful candidates as targets for new drugs. The gene coding for FtsH protease in $M$. tuberculosis has now been identified, cloned and expressed (Anilkumar et al 1998). The protein bears homology to FtsH proteins from other bacterial genera, although there are differences, and is thus a suitable site for directing a drug. Similar to $\mathrm{FtsH}$, the protein FtsZ is an analogue of eukaryotic tubulin. The FtsZ gene of $M$. tuberculosis has now been cloned and expressed (Ajitkumar, personal communication) and is also a suitable target for a drug.

\section{The present picture and future prospects}

The two key factors behind effective public health measures to combat TB are the prompt identification of infected individuals and a rapid laboratory confirmation of the infection. An epidemiological model (Murray and Salomon 1998) predicts that there will be a total of 225 million new cases and 79 million deaths from TB between 1998 and 2030. Active screening by sputum examination combined with a single contact treatment with $\mathrm{INH}$ could avert 24 million cases and lower mortality by nearly $40 \%$. More attention needs to be paid to the behaviour of patients as also the health provider. There is often considerable delay on the part of the physician in making a prompt diagnosis and making drugs available to the patient at the first point of contact. It has been reported that $80 \%$ of the chest symptomatics who reported to the nearest health facility did not get adequate and timely diagnosis; at times the delay in diagnosis was as long as 35 days. The same study also revealed that $75 \%$ of the chest symptomatics were simply turned away without subjecting them for sputum examination.

The control of TB at the population level depends to a large extent on the improvement of social, 
environmental and educational conditions. The health of slum dwellers of industrial Europe began to improve with the introduction of clean water supplies, sewage disposal and hygiene education in the late 19th century, when there were almost no effective medicines. It is well known that industrial smoke, as also smoke from active or passive smoking, weaken the lungs and make them susceptible to bacterial infection. In India an increase in the extent of female literacy is crucial for the improvement of public health because it turns out that whether women are literate or not influences acceptance of hygienic measures in the entire family. Incidentally, it is ironic that young females, in general, are more susceptible to infection by M. tuberculosis than young males of the same age.

Given a degree of fallibility in public health measures, research into drug-based and clinical therapies will have to continue. However, the production of a new drug which is ready for clinical use in India poses a serious financial problem. It has been recently reported [Nature (London) 395 $835,1998]$ that the introduction of a new drug in the USA involves an overall initial expenditure of $\$ 500$ million. At the current rate of exchange this is only marginally less than the sum of Rs 2988 crore which is the annual budgeted outlay by the Government of India for Science, Technology and Environment during 1999-2000!

Selection for drug-resistant strains is developing into a major problem and the population biology of Mycobacterium will need to be understood much better. Leach et al (1995) have shown that antibiotic resistant strains of Pneumococcus disappeared after the antibiotic-caused selection was removed. A possible explanation that has been put forward is that in the absence of antibiotics, a resistant strain is at a selective disadvantage relative to the wild type. If this is generally true with microorganisms, an intermittent antibiotic therapy may be better in the long run than a continuous therapy.

In view of the difficulties faced by developing countries, including India, to effectively treat cases of TB with drugs, and also because of the toxicity of the available drugs in certain cases, the use of a vaccine to prevent the onset of the disease appears to offer the best prospect for controlling TB world-wide. The model referred to above predicts that a vaccine with $50 \%$ efficacy would reduce by 36 million the expected number of new TB cases between now and 2030 and prevent 11 million deaths. The development of a more effective vaccine than BCG, which works well on infants and young children, has to await a clearer understanding of the virulence of $M$. tuberculosis and the immunology of the infection (S Vijaya, in preparation).

Curiously, during the same period in which the situation with regard to TB has worsened, the incidence of leprosy, caused by the related organism $M$. leprae, has registered a steep fall - according to a WHO report, the drop has been from 12 million symptomatic individuals worldwide to 1.2 million over the last 20 years. By strictly adhering to the triple drug regimen of dapsone, isoniazid and rifampicin for extended periods, the incidence of leprosy has been drastically reduced world-wide, and the disease may disappear from India in the course of the next few years (by 2002, according to a WHO forecast). This may be an overly optimistic view, but it remains a fact that chemotherapy has brought down the prevalence of leprosy in India significantly, from 20 per 10,000 in 1981 to 2 per 10,000 in 1997 (Sengupta 1999). Perhaps we can take a leaf from the methods and precautions taken to control leprosy to make the fight against TB too a success. These include simultaneous treatment with at least three different drugs and continuation of treatment till smear microscopy of sputum shows absence of organisms.

The alliance between TB and AIDS is emerging as a serious problem. HIV is thought to have entered India in the early 1980s, the first case being reported in March 1986. The results of several sero-surveillance surveys in different parts of the country have helped to provide an insight into the main modes of HIV transmission as well as identification of high risk groups within society. The 
profile of the epidemic varies widely from one region to another, reflecting the country's great diversity. Official estimates indicate that 1.5 million persons had become infected by HIV by the end of 1994 and this number is expected to go up to 2 million by the year 2000 . There is no mystery about the powerful effect of HIV infection on the natural history of TB. The link between the two has to do with the well established, though not widely appreciated, fact that $90 \%$ of the otherwise healthy persons infected. with $M$. tuberculosis do not develop clinical TB. These persons carry the infection but do not display the disease. Progression from infection to disease is ordinarily prevented by an intact immune system, particularly by cell-mediated immunity; and it is the immune response that is primarily compromised by HIV infection. A study undertaken in 1991 by the TB Research Centre, Madras, shows that the average risk of developing TB among HIV positives was as high as $2 \%$ per year; and $67 \%$ of the cases were detected within 30 months of registration. It is unfortunate that the funds available for controlling TB, both nationally and internationally, have been seriously affected in recent years by being partly siphoned off for the control of HIV (and also malaria).

Because of the HIV-TB nexus, it becomes important and urgent that the highest priority be given to further strengthening the TB programme in India. A portent of what can happen otherwise can be seen from the situation in the USA. From 1953 to 1984 the number of TB cases reported in the USA dropped by an average of 6\% each year. In contrast, from 1984 to 1997 there was an overall increase of $14 \%$ in new cases. Part of this reversal in a trend of long duration is undoubtedly due to the HIV epidemic: not only have the areas most affected by HIV been the same ones that have reported the largest increase in TB cases, the largest increase in TB cases has occurred among people in the age group ( 25 to 44 ) most affected by AIDS.

Finally, the question: Where do we stand now? We started at the 'foot of the ladder' prior to 1950 when there was no treatment for TB and went up from then to a maximum around 1984 during which time streptomycin and other antitubercular drugs were discovered. Since then, it has been a gradual fall due to drug resistance and HIV infection to a low 'rung in the ladder'. Whether we can go up the ladder again and how far depends on the efficacy of the methods followed in future in pursuit of this goal. The methods would include simultaneous treatment by at least three, preferably four, drugs for which the infective organism is sensitive, continuation of the therapy without break till smear microscopy shows the absence of the infection, and finally, control in the spread of HIV. For the authors, there is the disheartening realization that in spite of their best efforts and those of their colleagues, the aims with which they began almost 40 years ago have not been realized. Tuberculosis is prevalent in this country almost to the same extent as when they started their work. Hopes for ending this pathetic state of affairs rest on the development of a vaccine and the adherence by physicians and patients to a scientific attitude. And, equally important, simultaneously the government has to strengthen public measure programmes like control of pollution both in air and water, provision of safe drinking water and hygienic sewage disposal as well as promoting hygiene education among the masses, particularly among women, in order to make a success of the medical efforts to control tuberculosis.

\section{Acknowledgements}

We wish to thank M S Shaila and S Vijaya for their assistance in the preparation of the manuscript, to Mrs Sudha S Murthy for her guidance and active support and to our erstwhile colleagues and staff of the TB Sanatorium, Madanapalle, NTI, Bangalore and Indian Institute of Science, Bangalore for their support to us in the course of our work. We are grateful to $\mathrm{Dr} B \mathrm{~V}$ Venkatesha Murthy for the information on Ayurveda. Above all, we wish to acknowledge the assistance of our students whose queries and interactions with us have stimulated us to grow intellectually. 
Anilkumar G, Chauhan M M and Ajitkumar P 1998 Cloning and expression of the gene coding for FtsH protease from Mycobacterium TB $\mathrm{H}_{37} \mathrm{Rv}$; Gene 214 7-11

Bloom B R (ed.) 1994 TB: Pathogenesis, Protection and Control (Washington DC: Am. Soc. Microbiol.)

Chandrasekhar P 1988 Primary health care and TB programme; NTI Newslett. 24 1-23

Chauhan M M 1997 Reliability and applicability of serological tests in the diagnosis of TB; NTI Bull. 33 $56-61$

Cole S T, Brosch R, Parkhill J, Garnier T, Churcher C, Harris D, Gordon S V, Eiglmeier K, Gas S, Barry III C E, Tekaia F, Badcock K, Basham D, Brown D, Chillingworth T, Connor R, Davies R, Devlin K, Feltwell T, Gentles S, Hamlin N, Holroyd S, Hornsby T, Jagels K, Krogh A, McLean J, Moule S, Murphy L, Oliver K, Osborne J, Quail M A, Rajandream M-A, Rogers J, Rutter S, Seeger K, Skelton J, Squares R, Squares S, Sulston J E, Taylor K, Whitehead S. and Barell

B G 1998 Deciphering the biology of Mycobacterium TB from the complete gene sequence; Nature (London) 393 537-544; 396 190-198

Gayathri Devi B, Shaila M S, Ramakrishnan T and Gopinathan K P 1975 Purification and properties of peroxidase in Mycobacterium TB $\mathrm{H}_{37} \mathrm{Rv}$ and its possible role in the mechanism of action isonicotinic acid hydrazide; Biochem. J. 149 187-197

Heym B 1999 The molecular basis of isoniazid resistance in Mycobacterium TB; in Indo-French Symp. Multiple Drug Resistance and Emerging Diseases (New Delhi: Indian National Science Academy) Abstracts, p 10

Koch R 1932 Die Aetiologie der Tuberkulose; Am. Rev. Tuberc. 25 282-323 (Translated by Pruner B and Pinnor from the original 1882 article)

Leach A, Shelby-James T, Mayo M, Gratten M and Mathews J 1995 Report of a multidrug resistant clone of Steptococcus pneumoniae in aboriginal infants in the Northern Territory; Commun. Dis. Intelligence $19134-137$

Madhusudan K, Ramesh V and Nagaraja V 1994 Cloning and sequence analysis of DNA gyrase genes from Mycobacterium TB; Curr. Sci. 66 664-667

Madhusudan K and Nagaraja V 1996 Alignment and phylogenetic analysis of type II DNA topoisomerases; J. Biosci. $21613-629$

Mehta Gita 1997 Snakes and Ladders (London: Minerva)

Minnikin D E 1982 Lipids: Complex Lipids, their chemistry, biosynthesis and roles; in The Biology of Mycobacteria (eds) C Ratledge and J Stanford (London: Academic Press) vol 1 pp 95-184

Murray C J L and Salomon J A 1998 Modeling the impact of TB control strategies; Proc. Natl. Acad. Sci. USA 95 13881-13886

Ramakrishnan T 1983 Twenty five years of research on tuberculosis at the Indian Institute of Science, Bangalore; Curr. Sci. 52 398-402

Ratledge C and Stanford J (eds) 1982 The biology of mycobacteria vol. 1 (London, New York: Academic Press)

Rekha Prabhu, Ramananda Rao G, Jamaluddin M and Ramakrishnan T 1986 N-[2-naphthyl]-glycine hydrazide, a potent inhibitor of DNA-dependent RNA polymerase of Mycobacterium TB H37Rv; J. Biosci. $10163-166$ 
Riley M and Labadan B 1996 in Escherichia coli and Salmonella (ed) F C Neidhardt (Washington DC: Am. Soc. Microbiol.) pp 2118-2202

Rosen H, Richard H, Silver L, Kropp H, Dorso K, Kohler J, Sundelof J G, Huber J, Hammond G G, Jackson J J, Gill C J, Thompson R, Pelak B A, Epstein-Toney J H, Lankas G, Wilkening R R, Wildonger K J, Blizzard T A, DiNinno F P, Ratcliffe R W, Heck J V, Kozarick J W, and Hammond M L 1999 Reduced immunotoxicity and preservation of antibacterial activity in a released side-chain carbapem antibiotic; Science 283 703-706

Rozworski D A, Grant G A, Barton D H R, Jacobs W R Jr, Sachettini J C 1998 Modification of NADH of the isoniazid target (Inh A) from Mycobacterium TB; Science 279 98-102

Russell B 1969 The Autobiography of Bertrand Russell 1944-1969 (Simon and Schuster) p 16

Sengupta U 1999 Diagnosis of leprosy in India - Relevance and Perspectives; in Indo-French Symp. Multiple Drug Resistance and Emerging Diseases (New Delhi: Indian National Science Academy) Abstracts

Seshadri R, Suryanarayana Murthy P and Venkitasubramanian T A 1976 Isocitrate lyase in mycobacteria; Indian J. Biochem. Biophys. 13 95-96

Suryanarayana Murthy P S, Sirsi M and Ramakrishnan T 1973 Effect of age on the enzymes of tricarboxylic acid cycle and related enzymes in Mycobacterium TB H37Rv; Am. Rev. Resp. Dis. 108 689-690

Takayama K, Antony E L and David H L 1974 Restoration of mycolate synthetase activity in Mycobacterium TB exposed to isoniazid; Am. Rev. Resp. Dis. $11043-48$

Uplekar M W, Javekar S K and Parende S D 1996 TB management in private practice and its implications; Indian J. Tuberc. 43 19-22

Wang J C 1985 DNA topoisomerases; Annu. Rev. Biochem. 54 665-697

Waksman S A 1949 Streptomycin - Nature and Practical Applications (London: Bailliere, Tindall and Cox)

Wayne L G and Lin K Y 1982 Glyoxylate metabolism and adaptation of Mycobacterium TB to survival under anaerobic conditions; Infect. Immun. 24 363-370

Wilkinson E 1914 Notes on the prevalence of TB in India; Proc. R. Soc. Med. 8 195-225

Winder F G and Collins P B 1970 Inhibition by isoniazid of synthesis of mycolic acids in Mycobacterium TB; J. Gen. Microbiol. 63 41-48

Winder F G 1982 Mode of action of the antimycobacterial agents and associated aspects of the molecular biology of the Mycobacteria; in The Biology of the Mycobacteria (eds)

C Ratledge and J Stanford (London: Academic Press) vol 1, pp 353-438

World Health Organization 1996 Managing TB at national level. Global TB programme; (Geneva: WHO) pp 203-

210

World Health Organization Global TB Programme 1997 WHO Report on TB (Geneva: WHO) 